\title{
Challenges in Diagnosis and Treatment of Pediatric Multiple Sclerosis
}

\section{Desafios no Diagnóstico e Tratamento da Esclerose Múltipla Pediátrica}

Rita LOPES SILVA $\triangle 1$

Acta Med Port 2016 Jul-Aug;29(7-8):419-420 - http://dx.doi.org/10.20344/amp.8054

Keywords: Child; Demyelinating Autoimmune Diseases, CNS; Encephalomyelitis, Acute Disseminated; Multiple Sclerosis/diagnosis; Multiple Sclerosis/therapy.

Palavras-chave: Criança; Doenças Autoimunes Desmielinizantes do Sistema Nervoso Central; Encefalomielite Aguda Disseminada; Esclerose Múltipla/diagnóstico; Esclerose Múltipla/tratamento.

Pediatric multiple sclerosis (MS) is a rare disease, which has been increasingly recognized in the last years. It is defined by onset before the age of 18 years and was previously known as early onset MS and juvenile MS.

Pediatric MS is considered a multifactorial disease, in which the chronic inflammatory demyelination of the central nervous system (CNS) is caused by a combination of genetic vulnerability and environmental triggers. These triggers are still being investigated with controversial results, such as viral infections, inadequate exposure to sunlight, vitamin $D$ deficiency and exposure to cigarette smoke.

The global incidence of pediatric MS is unknown and few epidemiological studies exhibit diverse results from 0.5 to 0.9 per 100,000 . It is estimated that $3 \%-10 \%$ of all patients with MS will have onset before 18 and less than $1 \%$ before 10 years of age. ${ }^{1}$ The female preponderance is also seen in adolescent-onset MS, however prevalence is similar for female and male in early childhood-onset. ${ }^{1}$ A positive family history is present in $6 \%-8 \%$ of children with MS, moreover, parents and other relatives of pediatriconset MS patients are themselves at risk for typical MS onset.

The Portuguese multicentre study in pediatric MS that is now published involved 46 patients, ${ }^{2}$ recruited in 4 hospitals. In 6 cases the onset of disease occurred before 12 years of age and one patient has a positive family history of disease. The mean age at the first manifestation was 14 years and at confirmation of MS diagnosis was 16.1 years old.

Banwell et al published a systematic review of 1,540 pediatric MS patients, $50 \%-70 \%$ of them with a polyfocal presentation. ${ }^{1}$ The most frequent clinical manifestations were motor dysfunction (30\%), sensory dysfunction $(15 \%$ - $30 \%)$, brainstem symptoms $(25 \%)$, optic neuritis $(10 \%$ $22 \%)$ and ataxia $(5 \%-15 \%)$; isolated transverse myelitis occurred in less than $10 \% .{ }^{1}$ The first attack of the younger patients (less than 10 years) was more likely to display polyfocal deficits and altered behavior or consciousness, fulfilling criteria for acute disseminated encephalomyelitis (ADEM). Over $96 \%$ of these 1,540 pediatric patients had an initial relapsing-remitting course and relapses frequently occurred in the first $1-2$ years. ${ }^{1}$

According to International consensus clinical criteria, diagnosis of pediatric MS can be established in patients younger than 18 years with two episodes of CNS demyelination separated by more than 30 days and involving more than one area of the CNS, fulfilling clinical and radiological criteria of dissemination in time and space. ${ }^{3}$ The most recent revision of the McDonald criteria accepts MRI evidence of dissemination in a first typical clinical event; however in children less than 12 years of age these criteria are of less predictive value and should not be applied in ADEM like presentations. ${ }^{3}$

The MRI features of pediatric MS are quite similar to adult MS and involve the presence of multiple lesions, mostly in the white matter and typically observed in the periventricular area of the corpus callosum and spinal cord. ${ }^{4}$ Children often show more infratentorial lesions, predominantly in the pons, and may present large and tumefactive lesions with perilesional edema. ${ }^{4} \mathrm{~A}$ recommended minimum diagnostic panel for the initial inflammatory demyelinating event should define the disease burden with brain and cervical spinal cord MRI with and without gadolinium. ${ }^{5}$ The International Pediatric Multiple Sclerosis Study Group strongly recommended additional imaging of the entire spinal cord to identify demyelinating disorders with different treatment approaches. ${ }^{5}$

The diagnostic work-up should also include a cerebrospinal fluid (CSF) analysis, which is important to provide information regarding the inflammatory process, contributing to support the diagnosis and rule out other disorders that may display similar clinical or radiological findings. CSF cell count and protein are normal in almost $60 \%$ of pediatric MS patients, but the others present mild pleocytosis or increase in protein concentration. This diagnosis is supported by finding an increase in IgG index or oligoclonal IgG bands in over $90 \%$ of cases, although these can be absent in the presentation and only detected later in the disease course. The presence of oligoclonal bands

1. Pediatric Neurology Department. Hospital Dona Estefânia. Centro Hospitalar de Lisboa Central. Lisboa. Portugal.

$\triangle$ Autor correspondente: Rita Lopes Silva. ritalopessilva@hotmail.com

Recebido: 19 de julho de 2016 - Aceite: 19 de julho de 2016 | Copyright @ Ordem dos Médicos 2016 
in a first clinical CNS demyelinating event is correlated with evolution to MS.

At the initial presentation, it may be very difficult to distinguish pediatric MS from ADEM, which in a small subset of children may be associated with later development of MS, thus strengthening the importance of a prolonged follow-up.

As in adults, the differential diagnosis of pediatric MS, includes infeccious, inflammatory, vascular and neoplastic disorders, but additionally metabolic/genetic neurodegenerative disorders must be considered in this age group, namely mitochondriopathies, leukodystrophies and organic and aminoacidopathies. ${ }^{5}$

Pediatric MS can be considered a big challenge, since the younger children may display atypical clinical presentation (fever, encephalopathy, involvement of peripheral nervous system or other organ systems), absence or minimal resolution of symptoms, a progressive disease course, neuroradiological and laboratory features (elevated erythrocyte sedimentation rate, leukocytosis, marked CSF pleocytosis and absence of isolated CSF oligoclonal $\operatorname{lgG}){ }^{5}$ The presence of any of these features should lead to consideration of disorders other than MS. ${ }^{5}$

Such as in adult population, the first line treatment used for an acute demyelinating event is intravenous methylprednisolone pulse. ${ }^{6}$ If there is an incomplete response or in severe attacks, intravenous immunoglobulin and plasmapheresis should be considered. ${ }^{6}$ Since there are no randomized controlled studies in pediatric MS, the disease modifying therapies that intend to prevent relapses, are extrapolated from adult clinical trials and small retrospective, observational studies. ${ }^{6}$ The first line therapies are interferons or glatiramer acetate, with growing data supporting their efficacy and safety. In case of refractory disease, the use of natalizumab, daclizumab, cyclophosphamide and fingolomod are associated with increased risk of important side effects. ${ }^{6}$

A multidisciplinary team including pediatric and adult

\section{REFERENCES}

1. Banwell B, Ghezzi A, Bar-Or A, Mikaeloff Y, Tardieu M. Multiple sclerosis in children: clinical diagnosis, therapeutic strategies and future directions. Lancet Neurol. 2007;6:887-902.

2. Correia AS, Augusto L, Meireles J, Pinto J, Sousa AP. Pediatric multiple sclerosis in Portugal: a multicentre study. Acta Med Port. 2016:29:42531.

3. Krupp LB, Banwell B, Tenembaum S, International Pediatric MS Study Group. Consensus definitions proposed for pediatric multiple sclerosis neurologists, psychologist, nurse, social worker, physiotherapist and occupational therapist, is crucial to promote a high quality care for these young patients.

While adult neurologists are more experienced in following large cohorts of MS patients and regularly updated with the most recent clinical trials concerning preventive therapy, the pediatric neurologists feel more familiar with the differential diagnosis with infectious, metabolic and genetic neurodegenerative disorders in this age group and with the repercussions in school performance and behavior.

The cognitive dysfunction and psychological distress related to the direct neurologic impairment and the impact of the diagnosis of a chronic illness in adolescence should be recognized and may require an intervention in the functioning at home, school and among peers.

In my opinion "Pediatric Multiple Sclerosis in Portugal: A Multicentre Study"2 should be considered as an important first step towards a larger multicentre study, including more hospitals all around the country, where children and adolescents with MS are regularly followed.

The demographic, clinical, laboratorial and neuroradiological characteristics of the Portuguese patients are similar to those reported in previous studies. Concerning the comparison between the characteristics of childhood-onset MS (< 12 years) and adolescent-onset MS (12 - 18 years) it was found a male preponderance and CSF pleocytosis among the younger group. As expected, the analysis of the preventive treatments prescribed to pediatric MS patients in Portugal revealed a preference for interferons and glatiramer acetate.

The increase of awareness about pediatric MS and a closer collaboration between pediatric and adult neurologist centers would be a great opportunity to promote long-term studies in demyelinating disorders and to share knowledge, in order to improve the care of these children/adolescents and their families.

and related disorders. Neurology. 2007;68:S7-1.

4. Peña JA, Lotze TE. Pediatric multiple sclerosis: current concepts and consensus definitions. Autoimmune Dis. 2013:673947.

5. Hahn JS, Pohl D, Rensel M, Rao S, International Pediatric MS Study Group. Differential diagnosis and evaluation in pediatric multiple sclerosis. Neurology. 2007;68:S13-22.

6. Yeh EA, Weinstock-Guttman B. The management of pediatric multiple sclerosis. J Child Neurol. 2012;27:1384-93. 